SPACE RESEARCH COORDINATION CENTER

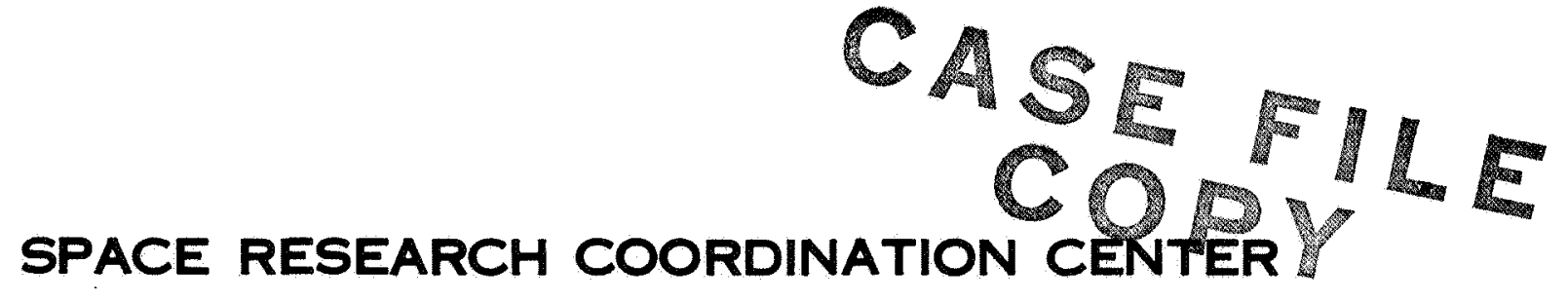

\title{
SIMULTANEOUS FLOW AND TEMPERATURE CORRECTION IN THE EQUILIBRIUM STAGE PROBLEM
}

\author{
BY \\ JOHN W. TIERNEY AND JOHN L. YANOSIK \\ DEPARTMENT OF CHEMICAL ENGINEERING
}

SRCC REPORT NO. 85

UNIVERSITY OF PITTSBURGH

PITTSBURGH, PENNSYLVANIA

7 OCTOBER 1968 
The Space Research Coordination Center, established in May, 1963, has the following functions: (1) it administers predoctoral and postdoctoral fellowships in space-related science and engineering programs; (2) it makes available, on application and after review, allocations to assist new faculty members in the Division of the Natural Sciences and the School of Engineering to initiate research programs or to permit established faculty members to do preliminary work on researchideas of a novel character; (3) in the Division of the Natural Sciences it makes an annual allocation of funds to the interdisciplinary Laboratory for Atmospheric and Space Sciences; (4) in the School of Engineering it makes a similar allocation of funds to the Department of Metallurgical and Materials Engineering and to the program in Engineering Systems Management of the Department of Industrial Engineering; and (5) in concert with the University's Knowledge Availability Systems Center, it seeks to assist in the orderly transfer of new space-generated knowledge in industrial application. The Center also issues periodic reports of space-oriented research and a comprehensive annual report.

The Center is supported by an Institutional Grant (NsG-416) from the National Aeronautics and Space Administration, strongly supplemented by grants from the A.W. Mellon Educational and Charitable Trust, the Maurice Falk Medical Fund, the Richard King Mellon Foundation and the Sarah Mellon Scaife Foundation. Much of the work described in SRCC reports is financed by other grants, made to individual faculty members. 


\title{
SIMULTANEOUS FLOW AND TEMPERATURE CORRECTION IN THE EQUILIBRIUM STAGE PROBLEM
}

\author{
John W. Tierney and John L. Yanosik \\ Chemical Engineering Department \\ University of Pittsburgh
}

Key words: A. Calculations-8, Temperature-6, 8, Flow Rate-6, 8, Material Balance-7, 8, 9. Compositions-9, Stages-9, Plates-9, Enthalpy Balance-7, 8, 9, Equations 10, Newton-Raphson Method-10, Equilibrium-9, Two-Phase System-9, Multicomponent-0, Columns-10, Distillation-10, Extraction-10, Stripping-10, Digital Computers-10, Matrices-10. 
ABSTRACT

Iterative methods for the solution of the steady state equilibrium stage problem are studied. Equations are first developed for calculating the effect of a change in temperature or flow rate on all energy balances, and then it is shown that these equations can be used in the multiple variable form of the Newton-Raphson correction process to correct either the temperatures or the flow rates when only energy balance errors are used. Similar equations for material balance errors which have been developed previously are then combined with the energy balance equations to provide a method for simultaneous correction of the temperatures and the flow rates. Because first order interactions between flow rates and temperatures are included the method is applicable to a wide range of equilibrium stage problems. Sample problems are presented, and it is shown that quadratic convergence is obtained for the simultaneous correction process. 


\section{INTRODUCTION}

The solution of the equilibrium stage problem is of both historical and practical importance to chemical engineers. Over the years many methods have been proposed--analytic solutions, graphical solutions, and numerical approximations. In the last few years there has been an emphasis on numerical methods because of the availability of large computing machines. In a previous

paper (1) it was pointed out that the computer solution is usually stated as that of finding a set of temperatures and flow rates which will satisfy all material and energy balances. For a system with $\mathrm{n}$ stages, there are $\mathrm{n}$ temperatures and $\mathrm{n}$ flow rates to be fixed, and there are $\mathrm{n}$ material balances and $\mathrm{n}$ energy balances to be satisfied. Direct solution is not possible, and iterative methods are used. At each step of the iterative process the material balance and energy balance errors are reduced by correcting the temperatures and flow rates. A linear correction process can be defined as:

$$
(\Psi)\left[\begin{array}{l}
C_{t} \\
C_{v}
\end{array}\right]=-\left[\begin{array}{l}
D_{m} \\
D_{e}
\end{array}\right]
$$

where $\left(C_{t}, C_{v}\right)$ is a vector containing the corrections to be made in the temperatures and flow rates, and $\left.\underset{m}{\left(D_{e}\right.}, D_{e}\right)$ is a vector containing the material balance errors and energy balance errors. is the correction matrix. There are many ways in which $\Psi$ can be defined, but in this paper it vill be the Jacobian matrix of the 
errors. It can be shown that the Jacobian matrix will give quadratic convergence in the vincinty of the solution, and this is the most rapid convergence possible with a linear correction process.

$\Psi$ can be partitioned into four nxn matrices,

$$
\underline{\Psi}=\left[\begin{array}{cc}
E_{t .} & E_{v} \\
J_{t} & J_{v}
\end{array}\right]
$$

It was shown in reference (1) that $\underline{E}_{t}$ is the only correction needed for the constant molal overflow problem and $\underline{E}_{v}$ is the only correction needed for the constant temperature extraction problem. Equations for $\underline{E}_{t}$ and $\underline{E}_{V}$ are summarized in Table 1 . Two minor modifications should be noted. One is that the Equation for $\underline{x}_{v}^{j}$ is written in a different but equivalent form. The second is that the normalization matrix $\mathrm{N}$ has not been included in the equation for $\underline{E}_{v}$. This point will be discussed later.

In this paper the equations for $\underline{J}_{t}$ and $\underline{J}_{\mathrm{v}}$ will be derived. It will then be shown that they can be used singly for certain problems or combined with $E_{t}$ and $\underline{E}_{V}$ to provide simultaneous quadratic convergence of temperatures and flows. Because $\mathrm{J}_{t}$ and $\mathrm{J}_{\mathrm{V}}$ are based on the energy balance errors, it is first necessary to obtain an expression for the energy balance and to define the error measures which will be used. 
TEMPERATURE CORRECTION USING ENERGY BALANCE ERRORS

If there is no shaft work, and if kinetic and potential energy effects are negligible, the energy balance error can be written in vector form as:

$$
D_{e}=\underline{L} H+\underline{V} G+Q+Q_{f}
$$

where $H$ and $G$ are liquid and vapor enthalpy vectors, $Q$ is the vector of heat exchange with the surroundings, and Qf is the vector of feed enthalpies. At the solution to the equilibrium stage problem, $D_{e}$ will be zero.

By definition of the Jacobian matrix, the elements of the kth column of $\underline{J}_{t}$ are obtained by differentiating Equation 3 with respect to the temperature in the $k$ th stage.

$$
\frac{\partial D_{e}}{\partial t_{k}}=\underline{L} \frac{\partial H}{\partial t_{k}}+\underline{V} \frac{\partial G}{\partial t_{k}}
$$

Varying $k$ from $l$ to $n$ will generate all the columns of $\underline{J}_{t}{ } \underline{H}_{t}$, and $\underline{G}_{t}$ are defined as the matrices whose $k t h$ columns are $H / \partial t_{k}$ ) and and $\left(\partial G / \partial t_{k}\right)$ respectively, then

$$
\underline{J}_{t}=\underline{L} \underline{H}_{t}+\underline{V} \underline{G}_{t}
$$

The effect of temperature on liquid and vapor enthalpy can be evaluated by expressing the enthalpies in terms of the 
partial enthalpies and then differentiating with respect to temperature

$$
\begin{aligned}
& H=\Sigma_{j}\left(\underline{H}^{* j} x^{(j)}\right) \\
& G=\sum_{j}\left(G^{* j} Y^{(j)}\right)
\end{aligned}
$$

where $\underline{H}^{* j}$ and $G^{* j}$ are diagonal matrices which have as elements the partial enthalpies of component $j$ in the liquid and vapor phases. The derivatives of Equation 6 and 7 with respect to $t_{k}$ are:

$$
\begin{aligned}
& \left.\frac{\partial H}{\partial t_{k}}=\Sigma_{j}\left(\underline{H}^{*}\left(\frac{\partial X(j)}{\partial t_{k}}\right)\right)+\Sigma_{j}\left(\frac{\partial H * j}{\partial t_{k}}\right) x^{(j)}\right) \\
& \frac{\partial G}{\partial t_{k}}=\Sigma_{j}\left(G^{* j}\left(\frac{\partial Y(j)}{\partial t_{k}}\right)\right)+\Sigma_{j}\left(\left(\frac{\partial G^{*}}{\partial t_{k}}\right) Y(j)\right)
\end{aligned}
$$

The first term in Equations 8 and 9 takes into account changes in enthalpy caused by changes in composition. The vector $\left(\partial x(j) / \partial t_{k}\right)$ is already known from the calculations for $\underline{E}_{t}$ and is the kth column of $x_{t}{ }^{j}$, defined by Equation Tl4 in Table 1. Similarly $\left(\partial Y(j) / \partial t_{k}\right)$ is the kth column of $\underline{Y}_{t}^{j}$ in Equation T15, Table 1 . The second term in Equations 8 and 9 accounts for changes in partial enthalpy due to changes in temperature at constant composition. It will be recalled that $\underline{H}^{j} \mathbf{j}$ and $\underline{G}^{* j}$ are diagonal matrices, and the kth diagonal element is the partial enthalpy on the kth tray. When the partial derivative with respect to $t_{\mathbf{k}}$ is 
taken, there will be only one non-zero term--on the kth row. The product of the derivative with $x^{(j)}$ will be a vector with only one non-zero term.

By varying $k$ from 1 to $n$ in equations 8 and 9 and assembling the vectors, it can be shown that

$$
\begin{aligned}
& \underline{H}_{t}=\Sigma_{j}\left(H^{j} * \underline{x}_{t}^{j}+\underline{M}_{3}^{j}\right) \\
& \underline{G}_{t}=\Sigma_{j}\left(\underline{G}^{* j} Y_{t}^{j}+M_{4}^{j}\right)
\end{aligned}
$$

where

$$
\begin{aligned}
& \underline{M}_{3}^{j}=\operatorname{diag}\left(m_{3^{\prime}}^{j}\right) ; m_{3}^{j}{ }_{i}=x_{i j}\left(\frac{\partial^{h}}{\partial t_{i}}\right) \\
& \underline{M}_{4}^{j}=\operatorname{diag}\left(m_{4}^{j}{ }_{i}^{\prime}\right) ; m_{4}^{j}{ }^{\prime}=y_{i j}\left(\frac{\partial^{g_{i}^{* j}}}{\partial t_{i}}\right)
\end{aligned}
$$

The partial derivatives in Equation 12 and 13 are fundamental properties of the materials themselves and are assumed to be known. For ideal solutions the derivatives are the best capacities of the pure components.

Substituting Equations 10 and 11 into Equation 5 gives the desired result for $\underline{J}_{t}$

$$
\left.\underline{J}=\underline{L} \Sigma_{j} \underline{(H *}^{* j} \underline{x}_{t}^{j}+\underline{M}_{3}^{j}\right)(\underline{N})+\underline{V}_{j}\left(\underline{G}^{* j} \underline{Y}_{t}^{j}+\underline{M}_{4}^{j}\right)(\underline{N})
$$


A normalization matrix has been included in Equation 14 to compensate for the fact that the mole fractions used in calculating $x_{t}^{j}, Y_{t}^{j}, M_{3}^{j}$, and $M_{4}^{j}$ do not sum to one. Normalizing the compositions should help, but experience to date has indicated that it has not made much difference in the problems tested. FLOW RATE CORRECTIONS BASED ON ENERGY BALANCE ERRORS

To obtain $\underline{J}_{v}$ it is necessary to differentiate Equation 3 with respect to the independent flow variables, holding temperatures constant. There can be only one independent flow variable per stage in variable flows. These variables are elements in the diagonal matrix $\underline{V}^{*}$. There is a corresponding matrix for the total liquid leaving each stage in variable flows, called $\underline{L}^{*}$. Through the overall material balance, $L^{*}$ can be expressed as a function of $\underline{V}^{*}$, so $\underline{L}^{*}$ is not an independent variable. The relation is given in Equation $T 21$, Table 1 , each element in $\underline{R}$ being the rate of change of one of the liquid rates with respect to one of the vapor rates. A more complete discussion of the definitions of the flow variables is given in reference 1 .

The kth column of $\mathrm{J}_{\mathrm{v}}$ will be obtained by differentiating Equation 3 with respect to the $k$ th independent flow variable, $v_{k}^{*}$,

$$
\frac{\partial^{D} e}{\partial v_{k}^{*}}=\underline{B}\left(\frac{\partial L^{*}}{\partial v_{k}^{*}}\right) H+\underline{L}\left(\frac{\partial \underline{H}}{\partial v_{k}^{*}}\right)+A\left(\frac{\partial \underline{V}^{*}}{\partial v_{k}^{*}}\right) G+\underline{V}\left(\frac{\partial^{L} G}{\partial v_{k}^{*}}\right)
$$

By varying $k$ from 1 to $n, I_{v}$ can be assembled. The result can be written as: 


$$
\underline{\mathrm{J}}_{\mathrm{V}}=\underline{\mathrm{B}}_{\underline{1}}+\underline{\mathrm{L}}_{\mathrm{V}}+\underline{\mathrm{W}}_{2}+\underline{\mathrm{V}}_{\mathrm{V}}
$$

By comparing Equation 15 with 16 the previously undefined matrices, $\underline{W}_{1}, \underline{H}_{\mathrm{V}}, \underline{W}_{2}$, and $\underline{G}_{\mathrm{V}}$ can be identified. Expressions for each of them will be developed in the next few paragraphs.

The columns of $\mathrm{W}_{1}$ are the product of the diagonal matrix $\left(\partial \underline{L}^{*} / \partial v_{k}^{*}\right)$ with the enthalpy vector $H$. It was mentioned above that the derivative of $\underline{L}^{*}$ with respect to vapor flow is given by $\underline{R}$. Hence, the general element of $\underline{W}_{1}$ is:

$$
\underline{w}_{1}+\left(w_{1} k_{i j}\right)=\left(r_{i j} h_{i}\right)
$$

The columns of $\mathrm{W}_{2}$ are obtained in a similar way, but the result is somewhat simpler because $\left(\partial \underline{v}^{*} / \partial v_{k}^{*}\right)$ is a matrix with only one non-zero element, the diagonal element in the kth row which is a $1: 0$. Using this plus the definitions for $\underline{A}$ and $G$, the general element of $\underline{w}_{2}$ is given by

$$
w_{2}=\left(w_{2}{ }_{i j}\right)=\left(a_{i j} g_{j}\right)
$$

Interpretation of $\underline{W}_{1}$ and $\underline{W}_{2}$ is as follows. Each element of $\underline{W}_{1}$ gives the change in enthalpy of the liquid leaving stage $i$ for a change of one unit in the vapor leaving stage $j$. Premultiplication by $\underline{B}$, which is the liquid flow connection matrix, will give a matrix for which the $i j$ element is the change in enthalpy of liquid phases entering and leaving stage $i$ for a 
unit change in vapor flow leaving stage $j$, with temperatures and compositions held constant. $\underline{W}_{2}$ gives the corresponding term for the vapor phases, and is simpler because the vapor phase flows are the independent variables. A term will appear in element $i j$ only if stage $i$ is the origin or destination of the flow $j$ which is being varied.

The other two terms in Equation 16 are $\mathrm{H}_{\mathrm{V}}$ and $\mathrm{G}_{\mathrm{v}}$, and are the changes in enthalpy per unit mass of material for a change in vapor flow at constant temperature. The only way that the enthalpy per unft mass can change under these conditions is through a change in composition. In terms of partial enthalpies

$$
\frac{\partial H}{\partial v_{k}^{*}}=\frac{x^{\Sigma} j^{H^{*}}{ }^{j}(j)}{\partial v_{k}^{*}}=\sum j^{H^{*}} \frac{\partial X^{(j)}}{\partial v_{k}^{*}}
$$

The effect of flow changes on composition is given by Equations T16 and T17 in Table 1. It then can be shown that

$$
\begin{aligned}
& \underline{H}_{v}=\Sigma_{j}\left(\underline{H}^{* j} \underline{x}_{v}^{j}\right) \\
& \underline{G}_{v}=\Sigma_{j}\left(\underline{G}^{* j} \underline{Y}_{v}^{j}\right)
\end{aligned}
$$

It will be noted that no normalization matrix is included in Equation 16. Although compositions are used in the calculation of $\underline{x}_{v}^{j}$ and $\underline{Y}_{v}^{j}$ examination of Equations $T 19$ and $T 20$ shows that the liquid composition involved in $M_{1}$ is that for the row, while the 
vapor composition involved in $\mathrm{M}_{2}$ is that for the column. No simple normalization can be made, and the equations from reference 1 which included this term have been modified in Table 1.

\section{SELECTION OF INDEPENDENT VARIABLES}

In the equations developed in the previous sections, the stage temperatures, interstage flows, and phase compositions have been the dependent variables. All other variables are independent and must be fixed before a solution is possible. An interchange of an independent and dependent variable can be made provided the column in the Jacobian matrix which corresponds to the dependent variable being removed is changed to accommodatethe new variable. One very useful interchange which is used in one of the sample problems is to fix the vapor flow leaving a stage, and to let the heat duty to that stage become a dependent variable. The change in the Jacobian matrix is simple in this case. The entire column corresponding to the flow being fixed is set equal to zero except for the element in the row corresponding to the energy balance for the stage, and this element is set to 1.0. In the correction procedure, the change in heat duty to the stage will be determined, holding the vapor flow constant.

\section{SOLUTION OF SAMPLE PROBLEMS}

The use of the equations derived in the last section for the solution of some sample problems will be discussed here. Results will be presented in terms of the Euclidean norm of the ersor vectors, which is defined as: 


$$
\begin{aligned}
& D_{m}=\left(\Sigma_{i}\left(d_{m, i}\right)^{2}\right)^{1 / 2} \\
& D_{e}=\left(\Sigma_{i}\left(d_{e, i}\right)^{2}\right)^{1 / 2}
\end{aligned}
$$

1. Stripper-Absorber Problem. In order to test $J_{t} a$ four-stage, six component absorber problem described by ilanson (2) as problem 7 was solved by using the correct flow rates in all iterations and using $\underline{J}_{t}$ to correct the temperatures. $\underline{J}_{v}$ was tested by using the same problem but fixing the temperatures in all iterations and solving for the flow rates. Results are presented in Table 2, and it can be seen that convergence is rapid and quadratic. The problem was also solved using simultaneous flow and temperature correction, and this of course required the calculation of the complete Jacobian matrix. Results are also summarized in Table 2 , and behavior is very similar to that with either flow or temperature correction alone. Hanson, using the same starting values, required 30 iterations to reduce $D_{e}$ to 31 and $D_{m}$ to $9.6 \times 10^{-5}$, not quite as good as obtained here in two iterations.

2. Distillation Problem. The second problem is a sixteen stage column with five components. It has been studied by Amudson (3) and Wang and Henke (4). They both used an alternating method in which the temperatures were corrected by the material balances errors and the flows by the energy balance errors. In the problem statement, the vapor product flow rate is fixed, and the condenser duty is to be determined. The results in Table 3 were obtained using the change of variable described in the last section, and 
it is evident that quadratic convergence is obtained. The material balance error at the third iteration in Table 3 is roughly equivalent to that obtained by Amudson at the tenth iteration and by Wang and Henke at the fifth iteration. More significant than the number of iterations is the rate of convergence near the solution. The simultaneous method is improving by orders of magnitude, while the others converge much more slowly. The slow convergence is probably due to the effect of flow changes on the material balance equations, indicated by the fact that the flow rates obtained by the simultaneous method differed by as much as $5 \%$ from the other solutions.

3. Multiple columns. The last problem is a twelve stage distillation column with a four stage side stripper, described by Hanson (2) as problem 9. From Table 4 it can be seen that quadratic convergence is also obtained with more complicated flow patterns than the strict countercurrent ones considered previously. After three iterations, the errors are somewhat less than those reported by Hanson after 32 iterations. One additional flow variable must be specified in a two-column system. In Table 4 the liquid feed to the second column was treated as a fixed flow and entered in I'. Another method which gave quadratic con vergence but is not reported in Table 4 is to fix the ratio of the amount of liquid removed from the drawoff tray to the amount passing to the next stage. 
COMPUTATIONAL CONSIDERATIONS

The simultaneous correction method requires the estimation of the effect of every temperature and flow change on all material and energy balances. The use of matrix notation seems necessary, and it has been found desirable to organize the programming so that matrix handling subroutines are used for most calculations. The order of calculation of the Jacobian matrix is also important. All calculations for one component should be done before the next component is started. Careful examination of the equations will show that this can be done. Calculations can be ordered by components because. there is no reaction, and the partial enthalpies and equilibrium ratios are assumed independent of composition.

The flow chart used for the sample problems is shoin in Figure 1. Equation references are indicated where appropriate. The program was written for an IBM 7090 with 32,000 words of core storage, and problems with up to 25 stages and 10 components can be solved without auxiliary storage. A detailed description of the program can be found in reference 5. The time required is roughly proportional to the number of components and to the square of the number of stages. The four stage problem required about 3 seconds per iteration, and the 16 stage problems about 40 seconds per iteration.

\section{CONCLUSIONS}

The multivariable form of the Newton-Raphson iteration method can be successfully used in the solution of the steady- 
state equilibrium stage problem. Equations have been developed for calculation of the partial derivatives needed in the Jacobian matrix, and it has been shown that the same equations can be used to solve a wide variety of problems--distillation, extraction, absorption, and stripping. It has not been necessary in the problems studied to date to place limitations on the size of the corrections, although this might be a useful technique if the starting values are far from the solution. The flow patterns need not be countercurrent, and it has been shown that interchange of variables permits some flexibility in the problem statement, while still maintaining quadratic convergence.

In all the problems studied to date, the Jacobian matrix has been calculated for each iteration. This gives the fastest convergence, but it is not likely that it is the most efficient from the stanpoint of computation. It has been observed that in the vicinity of the solution, the Jacobian matrix changes little, so that several iterations using the same correction matrix would seem to be a logical procedure. The utility of including interstage interactions in the correction proeess seems to be clearly established, but more work is needed to define the most efficient computational methods.

ACKNOWLEDGMENT

The authors gratefully recognize assistance in the preparation of this work from the National Aeronautics and Space Administration for a Traineeship and from the Computation and Data Processing Center at the University of Pittsburgh which received partial support under National Science Foundation Grant G-11309. 
Figure 1

Flow chart for simultaneous temperature and flow correction. Numbers in parentheses refer to equations in the text. ?.: 
NOTATION

Matrices are indicated by bold face symbols, vectors by upper case symbols, and scalars by lower case symbols.

$$
\begin{aligned}
& \text { A = Vapor recycle: } a_{i j} \text { is fraction of total vapor leaving } \\
& \text { stage } j \text { that goes to stage } i \text { if } i \neq j \text {, and } i s-1 \text { when } \\
& i=j \text {. } \\
& \text { B = Liquid recycle: } b_{i j} \text { is fraction of total liquid leaving } \\
& \text { stage } j \text { that goes to stage } i \text { if } i \neq j \text {, and is }-1 \text { if } l=j \text {. } \\
& c_{t^{\prime}} c_{v}=\begin{array}{l}
\text { Temperature and flow corrections: } c_{t, i} \text { and } c_{v, i} \text { are the } \\
\text { temperature and flow corrections for } s \text { tage } i .
\end{array} \\
& D_{m}, D_{e}=\text { Material and energy balance errors: } a_{m, i} \text { and } d_{e, i} \\
& \text { are the material and energy balance errors for stage } i \text {. } \\
& \underline{E}_{t}, \underline{E}_{v}=\text { submatrices of Jacobian matrix : } \\
& e_{t, i j}=\left(\partial d_{m, i} / \partial t_{j}\right) ; e_{v, i j}=\left(\partial d_{m, i} / \partial v_{j}^{*}\right)
\end{aligned}
$$

F, $F(j)=\begin{aligned} & \text { Feed matrix and } j \text { tin column of } \Gamma: f_{i j} \text { is the anount of } \\ & \text { component } j \text { in feed to stage } i .\end{aligned}$

$\mathrm{G}=$ Vapor enthalpy: $g_{i}$ is enthalpy of a unit mass of vapor

$G^{*} j=$ Partial enthalpy of vapor (diagonal) $: g_{i}^{* j}$ is the partial enthalpy of component $j$ for vapor in stage $i$.

$G_{t}=$ Temperature dependence for vapor enthalpy: $g_{t, i j}=\left(\partial g_{i} / \partial t_{j}^{*}\right)$

$\mathrm{G}_{\mathrm{v}} \quad=$ Flow dependence for vapor enthalpy: $g_{v, i j}=\left(\partial g_{i} / \partial v_{j}^{*}\right)$

II $=\begin{aligned} & \text { Liquid enthalpy: } h_{i} \text { is enthalpy of one unit mass of } \\ & \text { liquid in stage } i .\end{aligned}$

$\underline{H}^{* j}=$ Partial enthalpy of liquid (diagonal) $: h^{* j}$ is the partial enthalpy of component $j$ in stagei?.

$\underline{H}_{t} \quad=$ Temperature dependence for liquid enthalpy: $h_{t, i j}=\left(\partial h_{i} / \partial t_{j}\right)$

$\underline{\mathrm{H}}_{\mathrm{V}} \quad=$ Flow dependence for liquid enthalpy: ${ }^{\mathrm{h}} \mathrm{v}, \mathrm{ij}=\left(\partial \mathrm{h}_{i} / \partial v_{j}\right)$

$\underline{J}_{t} \underline{J}_{v}=$ submatrices of $: j_{t, i k}=\left(\partial d_{e, i} / \partial t_{k}\right) ; j_{v, i k}=\left(\partial d_{e, i} / \partial v_{k}^{*}\right)$ 


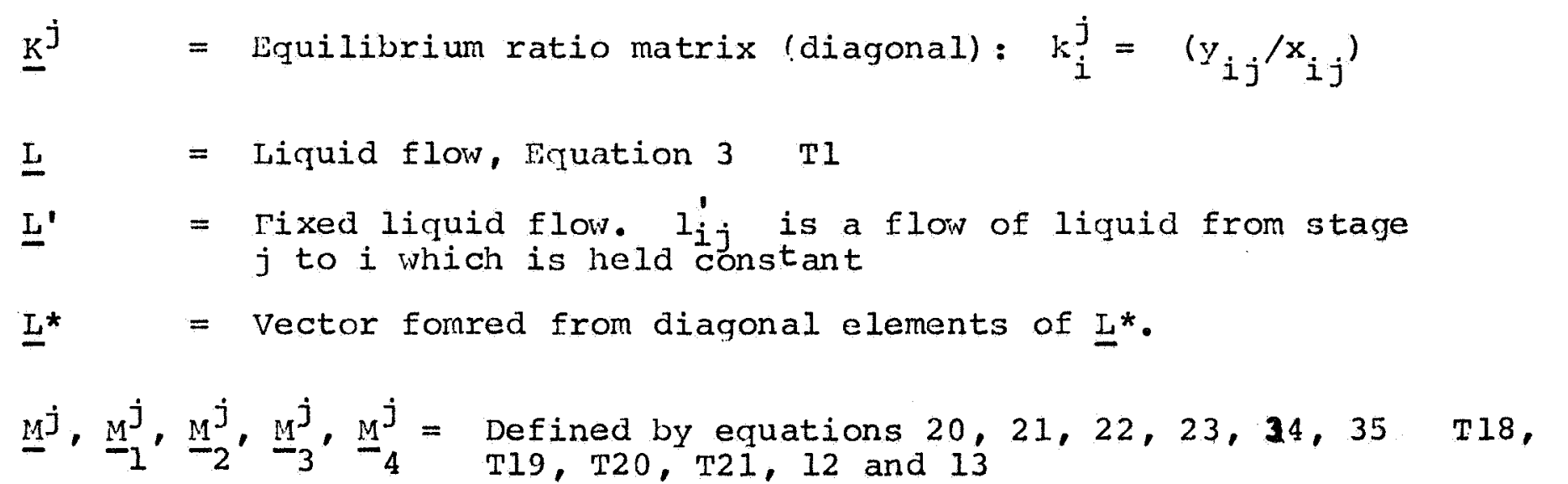

N $=$ Normalization matrix (diagonal), Equation 24 T22

$\Omega=$ Heat additions: $q_{i}$ is heat energy added to stage $i$.

$Q_{f} \quad=\begin{aligned} & \text { Feed enthalpy } \\ & \text { stage } i\end{aligned} q_{f, i}$ is total enthalpy of all feeds to

R = Defined by Equation 23 T21

$\mathrm{T}=$ Temperature: $t_{i}$ is temperature of stage $i$.

$\mathrm{U}=\mathrm{A}$ vector consisting of all ones.

V $\quad=$ Vapor flow, Equation $4 \quad \mathrm{~T} 2$

$\underline{V}^{\prime} \quad=$ Fixed vapor flow: $v_{i j}$ is a flow of vapor from stage $j$ to stage $i$ which is held constant.

$\underline{V}^{*} \quad=$ Vapor flow variable (diagonal): $V_{i}^{*}$ is the sum of all vapor flows leaving stage $i$ except ${ }^{1}$ those in $\underline{V}$.

$\mathrm{V}^{*} \quad=$ Vector formed from diagonal elements of $\mathrm{V}^{*}$.

$\underline{W}_{1}, \underline{W}_{2}=$ Defined by equations 39 , and $40 \quad 17,18$.

$\underline{x}, x^{(j)}=$ Temperature dependence for liquid composition: $x_{t, i k}^{j}=\left(x_{i j} / t_{k}\right)$

$\underline{x}_{v}^{j}=$ Flow dependence for liquid composition: $x_{v, i k}^{j}=\left(x_{i j} / v_{k}^{*}\right)$

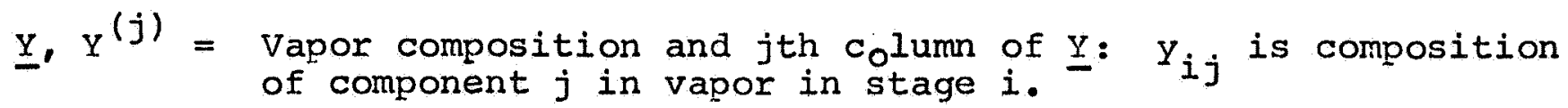
$\underline{y}_{t}^{j} \quad=$ Temperature dependencefor vapor composition: $y_{t, i k}=\left(\partial y_{i j} / \partial t_{k}\right)$ 
$\underline{Y}_{V}^{j}=$ Flow dependence for vapor composition:

$\quad y_{v, i k}^{j}=\left(\partial y_{i j} / \partial v_{k}^{*}\right)$

$\underline{z}^{j} \quad=\underline{L}+\underline{V} \underline{K}^{j}$

GREEK LETTERS

$\gamma=$ subscript indicating iteration number

$\Psi \quad=$ Jacobian matrix of errors

\section{SUBSCRIPTS}

$\therefore$ e $\cdot=$ Energy balance

$f \quad=$ Feed

$i, j, k=$ Indices for matrices or vectors.

$\mathrm{m}=$ Material balance

t. $1=$ Temperature

$=$ Flow

\section{SUPERSCRIPTS}

$j \quad=$ component

\section{REFERENCES CITED}

1. Tierney, J.W. and J.A. Bruno, A.I.Ch.E. Journal, 13, 556563 (1967).

2. Hanson, D. N., J. H. Duffin, and.G. F. Sommerville, "Computatio of Multistage Separation Processes", Reinhold, New York (1962).

3. Amundson, N. R.; and A. J. Pontinen, Ind. Eng. Chem., 50, 730-736 (1958).

4. Wang, J. C.: and G. E. Henke, Hydrocarbon Processing, 155-163 45 (1966).

5. Yanosik, John I., "Equilibrium Stage Calculations", M. S. Thesis, University of Pittsburgh (1967). 
Flow Matrices: $\underline{I}=\underline{B} \underline{L}^{*}+\underline{L}^{\prime}$

$$
\underline{\mathrm{V}}=\underline{\mathrm{A}} \underline{\mathrm{V}}^{*}+\underline{\mathrm{V}}^{\prime}
$$

Equilibrium Relationship: $Y^{(j)}=\underline{K}^{j} x^{(j)}$

Component Material Balance: $\left(\underline{L}+\underline{V} \underline{K}^{j}\right) x^{(j)}=-F^{(j)}$

$$
\underline{z}^{j} x^{(j)}=-F^{(j)}
$$

Overall Material Balance:

$$
(\underline{L}+\underline{V}) U+\underline{F} U=0
$$

Correction Vectors: $C_{t}={ }^{(T)} \gamma+1-(T)_{\gamma}$

$$
c_{v}=\left(V^{*}\right) \gamma+I-\left(V^{*}\right) \gamma
$$

Material Balance Error Measure: $D_{m}=(\underline{X}-\underline{Y}) U$

Correction Equations:- $E_{t} C_{t}+E_{v} C_{v}=-D_{m}$

$$
\underline{J}_{t} C_{t} \cdot \underline{I}_{v} C_{v}=-D_{e}
$$

Jacobian Matrices $E_{t}$ and $E_{v}$

$$
\begin{aligned}
& \underline{E}_{t}=\Sigma\left(\underline{x}_{t}^{j}-\underline{Y}_{t}^{j}\right) \underline{N} \\
& \underline{E}_{v}=\sum_{j}\left(\underline{x}_{v}^{j}-\underline{x}_{v}^{j}\right) \\
& \underline{x}_{t}^{j}=-\left(\underline{z}^{j}\right)^{-1} \underline{v} \underline{M}^{j} \\
& \underline{y}_{t}^{j}=\underline{M}^{j}+\underline{\underline{K}}^{j} \underline{x}_{t}^{j} \\
& \underline{x}_{v}^{j}=\left(\underline{z}^{j}\right)^{-1}\left(B \underline{M}_{I}^{j}-\underline{M}_{2}^{j}\right) \\
& \underline{y}_{v}^{j}=\underline{K}^{j} \underline{x}_{v}^{j} \\
& \underline{M}^{j}=\operatorname{diag}\left(\frac{d k_{1}^{j}}{d t_{1}} x_{1 j}, \ldots \frac{d k_{j}^{j}}{d t_{i}} x_{i j}, \ldots\right) \\
& \underline{\underline{M}}_{1}^{j}=\left(m_{1, i k}^{j}\right)^{d t_{1}}=\left(r_{i k}{ }^{\prime} x_{i j}\right) \\
& \underline{M}_{2}^{j}=\left(m_{2}^{j}, i k\right)=\left(a_{i k} Y_{k j}\right) \\
& \underline{R}=\underline{\underline{B}}^{-1} \underline{A}=\left(r_{i k}\right)=\left(\frac{\partial_{i}^{*}}{\partial v_{k}^{*}}\right) \\
& \underline{N}=\operatorname{diag}\left(1 /\left(_{j}^{\Sigma}\left(x_{1 j}\right)\right), \ldots \ldots 1 /\left(_{j}^{\Sigma}\left(x_{i j}\right)\right), \ldots\right)
\end{aligned}
$$

$$
\text { TABLE } 1
$$


Iteration

. Number
Temperature

Correction

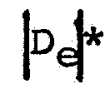

0

1

2

*
Flow Correction
Simultaneous Flow and Temperature Correctiol
$2.4 \times 10^{3}$
$2.5 \times 10^{2}$
$2.4 \times 10^{3}$
8.0
$1.1 \times 10^{2}$
$7.4 \times 10^{-3}$
$2.3 \times 10^{-1} \quad 1.8 \times 10^{-5}$
$1.0 \times 10^{2}$
$1.6 \times 10^{-1}$

pep *

$\left|D_{e}\right| *$
$\left|D_{m}\right| *$

Based on One Mole of Absorber Iiquid

TABLE 2

Error Measures for Sućcessive Iteration of Four stage Absorber Problem

Iteration

Number

0

1

2

3

4

5
Starting Values

Reference 3
$\left|D_{e}\right| *$ $4.1 \times 10^{3}$

$\left|p_{m}\right|$

$5.1 \times 10^{-1}$

$5.8 \times 10^{2} 1.3 \times 10^{-1}$

$2.9 \times 10^{1} 8.9 \times 10^{-3}$

$8.3 \times 10^{-2} 5.0 \times 10^{-5}$

\section{Starting Values}

Reference 4 
Iteration

Number

$|\mathrm{D} e|^{*}$

$\left|\mathrm{D}_{\mathrm{m}}\right|$ *

0

$$
1.3 \times 10^{4}
$$$$
6.2 \times 10^{-1}
$$

1

$2.5 \times 10^{3} \quad 3.0 \times 10^{-1}$

2

$3.6 \times 10^{2}$

$5.4 \times 10^{-2}$

3

$$
2.1 \times 10^{1}
$$

$1.5^{\circ} \times 10^{-3}$

4

$$
3.6 \times 10^{-2} \text {. }
$$$$
4.0 \times 10^{-6}
$$

* Based on one Mole of Feed

TABIE 4

Error Measures for Successive Iterations of Multiple Column Problem 